\title{
e-Phaïstos
}

e-Phaïstos

Revue d'histoire des techniques / Journal of the history

of technology

VI-2 $2017 \mid 2018$

La diversité du patrimoine technique africain

\section{Déséquilibre entre réutilisation et conservation du patrimoine industriel en Chine}

The Imbalance between Reuse and Conservation of Industrial Heritage in China

Jiali HUANG

\section{OpenEdition}

Journals

Édition électronique

URL : http://journals.openedition.org/ephaistos/3565

DOI : 10.4000/ephaistos.3565

ISSN : 2552-0741

Éditeur

IHMC - Institut d'histoire moderne et contemporaine (UMR 8066)

Référence électronique

Jiali HUANG, « Déséquilibre entre réutilisation et conservation du patrimoine industriel en Chine », ePhaïstos [En ligne], VI-2 2017 | 2018, mis en ligne le 16 novembre 2018, consulté le 02 mai 2019. URL : http://journals.openedition.org/ephaistos/3565; DOI : 10.4000/ephaistos.3565

Ce document a été généré automatiquement le 2 mai 2019.

Tous droits réservés 


\section{Déséquilibre entre réutilisation et conservation du patrimoine industriel en Chine}

The Imbalance between Reuse and Conservation of Industrial Heritage in China

Jiali HUANG

1 La Chine est un pays vaste mais très déséquilibré en termes de ressources naturelles et de développement économique. Pour des raisons historiques et géographiques, elle est beaucoup plus peuplée et développée économiquement à l'est sur le littoral qu'à l'ouest. Dans la région de l'Est, de grandes villes, qui étaient des ports commerciaux à l'époque coloniale, ont bénéficié de l'expansion industrielle des faits de l'investissement étranger et de la desserte, qui a facilité la circulation des matières premières, des marchandises et du savoir-faire. Aujourd'hui, la Chine connait trois degrés de développement: de vastes étendues rurales agricoles, de nouveaux centres industriels en cours d'industrialisation, et de grandes métropoles soumises à la désindustrialisation. En ce sens, la distribution géographique des vestiges industriels et le niveau de concentration sont par défaut déséquilibrés. Cela dit, à cause de la rapidité du développement, les grandes villes dotées de sites industriels en quantité sont confrontées dans un premier temps aux défis que constituent l'abandon et la reconquête de vastes terrains industriels. Pourtant, par comparaison avec l'Occident, ces derniers témoignent de cette période courte, intense et désordonnée d'industrialisation que la Chine a connue depuis la fin du $\mathrm{XIX}^{\mathrm{e}}$ siècle jusqu'à aujourd'hui, caractérisée par la rupture, la reprise et le progrès provoqués par les conflits nationaux, régionaux, et internationaux et les efforts chinois ${ }^{1}$. (Fig. 1) 


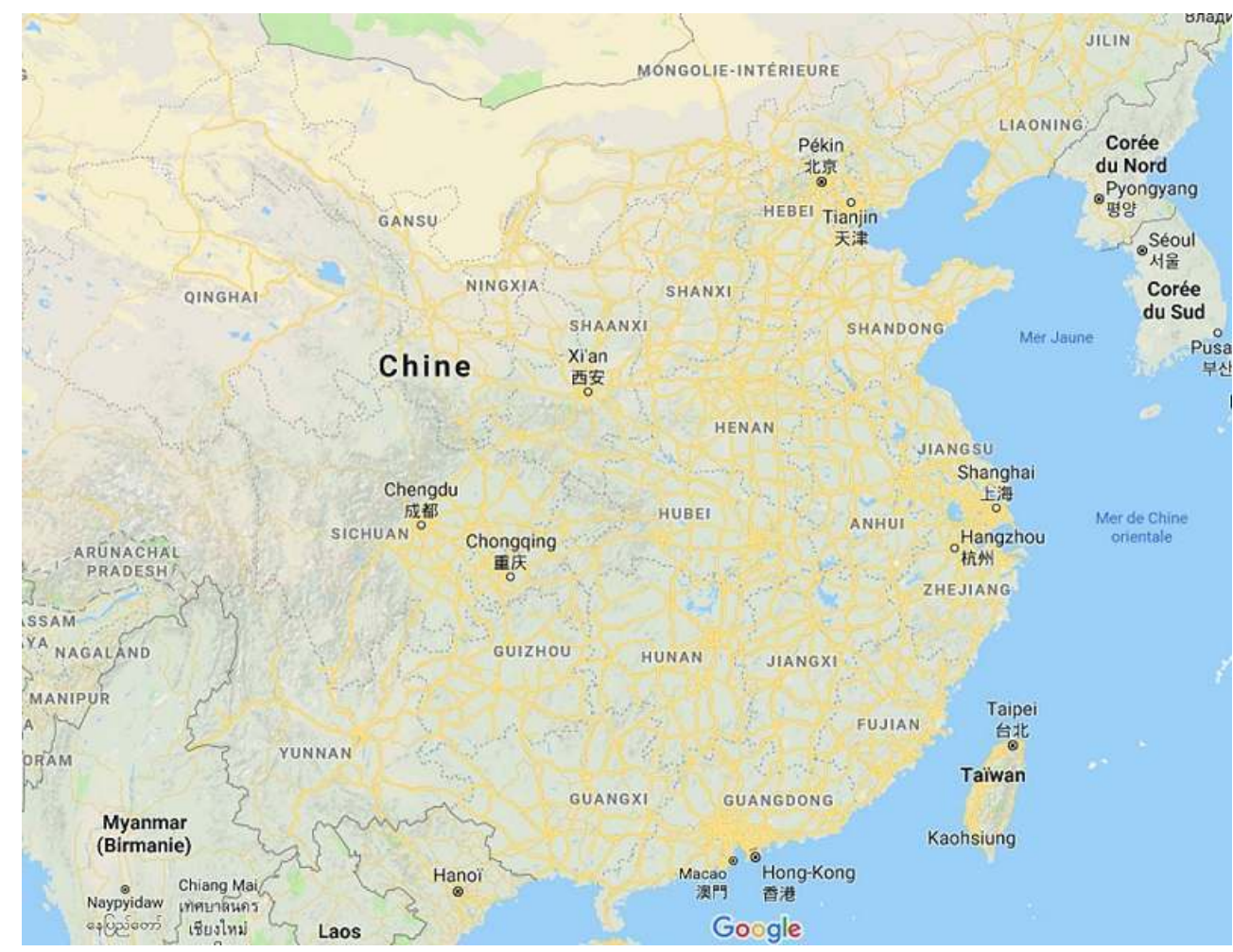

Données cartographiques @ 2018 Google, SK telecom, ZENRIN

2 De nos jours, il nous semble que le déclenchement de la patrimonialisation des territoires industriels après la désindustrialisation suit le même chemin : un phénomène immédiat de friches industrielles et une vague de reconversions. Il faut préciser que l'explosion des réhabilitations, dont la plupart en parc créatif fortement répétitif, est un phénomène unique dans les grandes métropoles chinoises telles que Pékin, Shanghai, Guangzhou etc. Le parc créatif est une forme nouvelle et unique d'espace consacré à l'accueil des entreprises dédiées à la culture et aux connaissances, soutenue par une autre politique après la stratégie de construction des parcs industriels. Ces deux formes préconisées par le gouvernement sont successivement considérées comme un nouveau volet de croissance économique aujourd'hui. La Chine n'est plus une société aussi fermée qu'elle l'était il y a cent ans, ni aux yeux des étrangers ni même dans l'esprit des Chinois. Bien que le développement de la recherche sur le patrimoine industriel soit en retard, on observe ces dernières années un essor des études réalisées par de jeunes chercheurs, qui ont été encouragés par des modèles de réaménagement de régions industrielles autrefois polluées et désertées. Un certain nombre d'entre eux se consacrent, non seulement à démontrer la valeur de ce patrimoine pour la société, mais aussi à trouver de bons moyens pour sa valorisation en participant à des projets de réhabilitation. Ces projets se sont réalisés à partir du présupposé que des bâtiments obsolètes recèlent un potentiel énorme de reconversion et peuvent répondre à de nouvelles demandes, ce qui n'est pas faux.

Mais ces discours sur la valeur exceptionnelle du patrimoine industriel, un concept préalable reconnu par l'UNESCO, et sur l'importance de sa conservation, paraissent largement insuffisants. Surtout la Chine est un État partie de la Convention du patrimoine 
mondial et parmi les états dotés de la plus longue liste des biens inscrits. Les conventions internationales de l'UNESCO sont reconnues par la Chine et les principes sur la conservation des sites patrimoniaux rédigés par l'ICOMOS Chine ont été mis en place pour guider les pratiques conformes à la situation chinoise ${ }^{2}$. Voici la définition du patrimoine culturel donnée par la loi des biens culturels:«les vestiges détenteurs de valeurs historique, artistique et scientifique, les architectures liées aux personnages et incidents historiques importants, les objets d'art et artisanaux ainsi que les spécimens naturels ». Cette définition intégrale tient compte de toutes les catégories de témoins historiques légués du passé, mais nous remarquons qu'elle se réfère plutôt à la richesse tangible et que l'accent dans les actions de conservation est mis sur les vestiges de l'époque ancienne. De ce fait, on tend à s'interroger sur les causes du déséquilibre entre les pratiques nombreuses de valorisation et le manque de préoccupation pour la sauvegarde de l'authenticité et de l'intégrité du patrimoine, et de même sur celui qu'on observe entre la transformation précoce en parc créatif et d'autres tentatives de reconversion. Cet article vise à analyser ce phénomène de déséquilibre, en tenant compte du contexte politique et urbain, et il révèle les raisons fondamentales de la méconnaissance du patrimoine industriel de la part des milieux académiques, législatifs et professionnels. Pour finir, en exposant les résultats insatisfaisants des parcs créatifs réalisés, nous proposerons des pistes possibles pour parvenir à un équilibre entre la conservation et la valorisation.

4 En moins d'une décennie, l'essor des reconversions des friches industrielles en parc créatif a été un épisode spectaculaire de l'urbanisation et la panacée pour un quartier industriel vieilli et délaissé, promu par le mouvement de Récession d'industrie lourde et introduction d'industrie tertiaire dans la ville ${ }^{3}$. Ce mouvement a entraîné le déménagement de nombreuses usines polluantes vers la banlieue, vidé celles qui étaient en cours de fermeture, et finalement rendu disponibles de nombreux terrains dispersés. En raison de leur dimension gigantesque, ces usines situées autour de petites villes y ont été intégrées au fur et à mesure par l'urbanisation et possèdent ainsi une valeur foncière accrue. À Guangzhou, la politique de réaménagement de la ville dégradée (2010-2020) met à disposition des terrains industriels d'une superficie de $137.16 \mathrm{~km}^{2}$, dont $41.6 \%$ seront occupés à des fins commerciales et résidentielles, $42 \%$ seront dédiés à l'administration, au bien-être, à la voirie et aux espaces verts, et $16 \%$ seront consacrés à l'écologie 4 .

5 Pour actionner le moteur économique et la régénération de la ville, celle-ci devrait s'appuyer sur de nouveaux piliers économiques et industriels dont l'interprétation se borne aux industries créatives. Les industries créatives sont une notion d'origine anglosaxonne et les premières définitions s'interprètent dans le sens d'une économie innovante liée aux services intangibles fournis par les talents, telles que l'informatique, la culture, l'art, les brevets, l'éducation etc. ${ }^{5}$ par rapport à la production manufacturière. Elles sont considérées comme une solution de relance économique plus prometteuse que les industries productives et tertiaires. Intégrées par des instruments politiques locaux, elles favorisent une nouvelle modalité d'entreprise qui génère une grande valeur ajoutée et de nouveaux emplois pour la requalification des grandes métropoles qui ont perdu la dynamique de développement. La région de la Ruhr en Allemagne, le quartier de Soho à New York, les Docklands à Londres sont des cas tellement classiques que des chercheurs chinois les citent toujours en vantant un environnement propre et agréable, ainsi qu'une économie puissante, générés par ces projets merveilleux. Impatients d'imiter ces modèles réussis, les bureaux d'urbanisme des grandes métropoles annoncèrent l'un après l'autre 
leur politique de transformation des friches industrielles en parc créatif, avec pour objectifs des centaines de projets et des milliards de yuans en retour 6 . On peut distinguer trois étapes dans leur réalisation et chacune se fait selon des modalités différentes.

À partir de la fin du $\mathrm{XX}^{\mathrm{e}}$ siècle, la transformation des usines a été lancée à l'initiative d'artistes d'avant-garde, qui cherchèrent à y installer leurs ateliers avec un loyer modeste, à l'instar de 798 à Pékin (un complexe de l'usine électronique 798), du M50 à Shanghai (magasins d'une usine textile le long de la Rivière Suzhou) et de la conserverie "rouge » de poisson à Guangzhou (à cause de l'architecture en briques rouges). Ils transformèrent les bâtiments par une création artistique libre selon leur goût, et ceci retint éventuellement l'attention du public et des médias, de sorte que les autorités commencèrent à s'engager à les soutenir par financement. Puis, le gouvernement poursuivit la mise en œuvre de plusieurs projets de réappropriation des bâtiments industriels et de nouvelles constructions avec des fonds financiers, projets dénommés parcs créatifs autorisés (PCA). La troisième étape se déroula avec la prise en conscience des propriétaires des usines des profits potentiels de la reconversion, et ils prirent en charge la planification, et l'évaluation jusqu'à l'achèvement du projet. La seconde phase de M50 se poursuivit par le biais de la planification et de la gestion d'une agence créée par l'usine, qui fut un grand succès. Selon un rapport sur le développement des industries culturelles et créatives en 2013, Shanghai détenait 118 PCA et la valeur produite par l'industrie culturelle atteignait $11,5 \%$ du PIB de Shanghai.

7 Outre la valeur économique engendrée et la création de nouveaux emplois, cette politique préconisée par le gouvernement est d'une certaine manière une force motrice de régénération urbaine. Elle change immédiatement l'image négative des friches industrielles en leur redonnant une nouvelle fonction au sein de la communauté qui vit et évolue aux alentours. Cette réinsertion est très importante pour le développement durable, qui est notre plus gros problème et défi aujourd'hui, puisqu'elle nous permet d'épargner l'énergie et les ressources exigées par de nouvelles constructions. Quant à la préservation du patrimoine, la réhabilitation assure la sauvegarde des sites industriels représentatifs de l'anéantissement entraîné par la grande vague de destruction et d'urbanisation. Il faut se rendre compte que les vestiges de la civilisation industrielle ne seront jamais récupérables une fois détruits et qu'une partie de la mémoire de la ville sera effacée. Déjà, nous avons beaucoup perdu dans la bataille pour la sauvegarde de la mémoire en voyant une si grande quantité de bâtiments anciens détruits par les bulldozers et les communautés chassées du lieu où les générations ont vécu. Ces destructions irrémédiables nous servent de leçon et nous font prendre conscience que le patrimoine est effectivement le gardien d'une mémoire collective, qui peut être un mode de vie, un esprit, un savoir-faire partagé par une communauté et qui est favorable à la pérennisation de sa culture et sa vitalité. Ce lien avec le passé existe toujours dans la tradition chinoise qui le considère comme sacré et transférable de générations aux générations, et les architectures, les formes artistiques ainsi que les pratiques coutumières en font témoins. Analogiquement, les traces et empreintes marquées par le passé d'industrialisation sont témoins de l'évolution du paysage et des infrastructures de notre ville ainsi que de la société apportée par l'industrie moderne. Incontestablement, la mémoire restera vive et les liens pourront être tenus si ces traces sont présentes.

8 Cette volonté de réutiliser des bâtis existants et les bénéfices patents des projets de reconversion doivent être appréciés. Cependant, des projets inappropriés ont des effets néfastes, qui nous amènent à réfléchir sur le décalage entre la réhabilitation d'envergure 
et l'absence de mesures de conservation. Par exemple, Tong Le Fang est un exemple d'agglomération datant des années 30 de petites fabriques localisées au sein d'un quartier de Nong Tang7 au centre à Shanghai. Malheureusement, le quartier occupé par des usages disparates a été mal entretenu jusqu'à la veille de la reconversion. Sa transformation a pour objectif une réhabilitation en parc créatif, qui finalement abrite des ateliers d'art, des agences de média ainsi que des services de loisirs. Nous remarquons les modifications majeures de l'extérieur des bâtiments qui s'éloignent de leur image historique et qui ne sont pas en harmonie avec l'environnement : adjonctions de structures en verre de style classique occidental, réfection des murs en jaune masquant l'ancienne apparence etc. (photos 1 et 2). La structure a été bien renforcée et l'apparence a été renouvelée. Ce sont les interventions sans tenant compte de l'authenticité qui sont jugées inappropriées pour la conservation. Cette réhabilitation constitue un cas de projet réussi selon des critères économiques plutôt que patrimoniaux. Nous remettrons donc en cause la relation inhérente entre réutilisation et conservation, en cherchant de bons moyens de réalisation des projets mieux adaptés à chaque cas spécifique. Sans doute, ce déséquilibre devra être examiné avec une observation des raisons à plusieurs niveaux: la politique urbaine, la recherche académique, le système juridique, qui sont les facteurs principaux de la sauvegarde des sites et du pilotage des projets.

Photo $n^{\circ} 1$. Exemple de réhabilitation d'un ancien complexe usinier à Shanghai

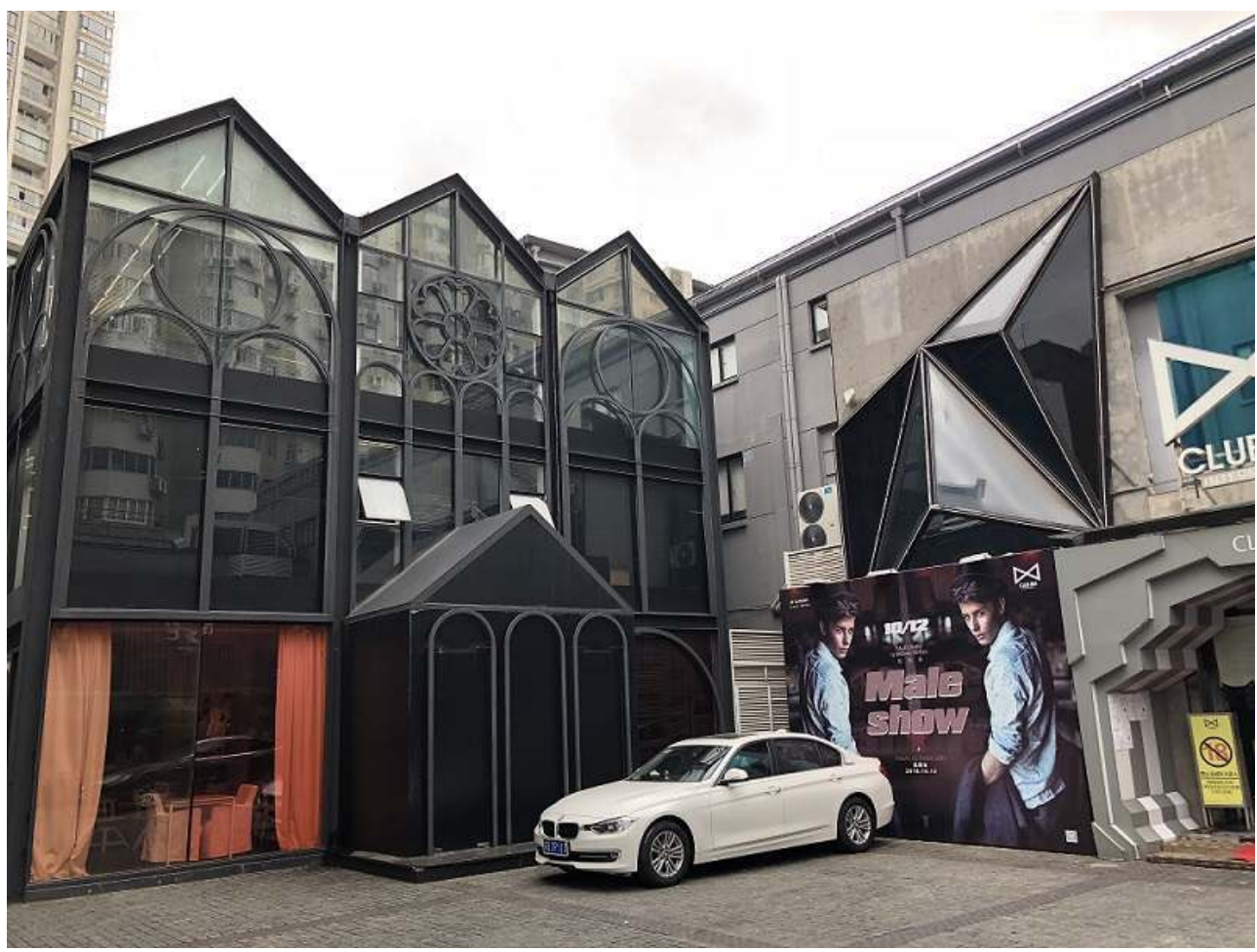

La structure en verre est incohérente avec le style des autres bâtiments

Crédit photographique : Zhuang Jiayan 
Photo $n^{\circ} 2$. Exemple de réhabilitation d'un ancien complexe usinier à Shanghai

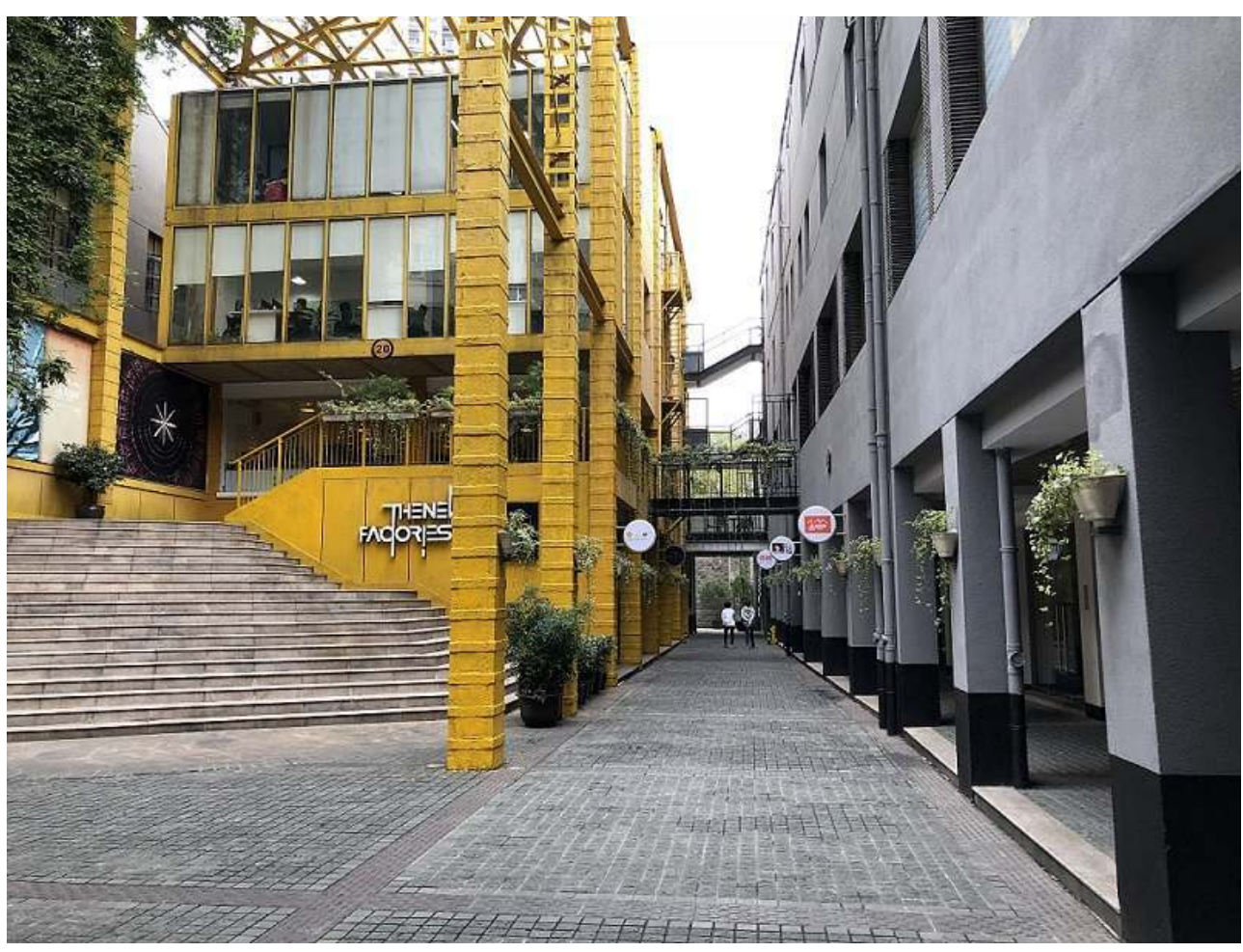

La peinture en jaune des bâtiments a dénaturé leur image originelle

Crédit photographique : Zhuang Jiayan

\section{Politique urbaine}

D'abord, les avantages obtenus par la politique en faveur des parcs créatifs comprennent une réduction de la valeur foncière, une réduction d'impôts, une baisse de la taxe sur des produits exportés, des aides financières et le privilège de recrutement de main d'œuvre qualifiée, afin d'encourager la création de nouvelles compagnies dans le secteur tertiaire. Incités par ces avantages, les parcs se forment à la hâte, abritant souvent une palette de composantes multifonctionnelles: agences de design, d'architecture, ou de médias, ateliers d'artistes, boutiques de décoration et quelques restaurants. Sans orientations plus précises et une planification fondée sur les avantages, les faiblesses, le positionnement et les fonctions prospectives, les parcs ne font que se répéter l'un l'autre en termes de modalités et d'apparence. Ils n'ont pas réussi à créer une ambiance favorable pour une véritable agglomération d'entreprises de qualité spécialisées en leur sein. Ensuite, à chaque étape mentionnée ci-dessus, ont surgi des problèmes aggravant la situation: manque de subventions pour des projets gouvernementaux à long terme ; initiatives individuelles disparates et reconversion médiocre entraînant une baisse de la qualité architecturale; compagnies immobilières n'hésitant jamais à entreprendre ces projets de transformation en visant une rentabilité de loyer dans le proche avenir. Au cours d'une enquête sur les projets de parcs créatifs à Guangzhou, j'ai observé que la plupart des bâtiments ont été transformés en bureaux pour de petites entreprises, restaurants et bars attirés par les loyers modérés. Même s'il y a des galeries d'art, elles se confondent avec d'autres fonctions, ce qui donne une impression de désordre. Confrontés à des loyers en 
augmentation constante exigés par l'agence de gestion, ces artistes sont obligés de partir. Par exemple, le loyer de la conserverie rouge est passé de 30 yuans $/ \mathrm{m}^{2}$ à 120 yuans $/ \mathrm{m}^{2}$ par rapport au loyer moyen de 58 yuans $/ \mathrm{m}^{2}$ pour les espaces de bureaux dans toute la ville, soit 8 euros $/ \mathrm{m}^{2}$. Dans ce cas, sans créer une valeur dite « industrielle », la rentabilité de ces parcs créatifs est recherchée par le biais du revenu des loyers et des petits commerces, et ils deviennent parcs « lucratifs » plutôt que « créatifs ». On constate que la presque totalité des espaces dans les parcs tels que Padi, l'ancienne brasserie de Zhujiang et ses environs, et Taigucang, les anciens magasins de Swire à Guangzhou, sont loués à des restaurants, bars et caves à vin, sauf pour le bâtiment principal de la brasserie transformé en musée.

Quand le parc créatif devient une solution universelle pour toutes les friches industrielles, il en résulte une concurrence défavorable entre ces parcs, qui fait baisser le taux de location. Prenons le cas de Guangzhou: si l'on y fait une comparaison des loyers en moyenne dans les parcs les plus connus, à part deux ou trois, il reste toujours des espaces à louer, ce qui ralentit ainsi la rentabilité. Inéluctablement, même pour la consommation, la dégradation de l'ambiance générale, le défaut de réponse aux attentes publiques, et le manque de bénéfices pour la communauté locale, sont les raisons essentielles de la chute d'intérêt, de fréquentation et d'implication des visiteurs. Selon l'enquête réalisée par Deng Yawen dans son mémoire ${ }^{8}$, les visiteurs ont tous la même impression sur les parcs créatifs de Guangzhou en les trouvant trop commerciaux, alors que leur principale intention de fréquentation était de se détendre et d'apprécier un paysage industriel imposant. Au contraire, ils ont apprécié la conserverie rouge de poisson, où davantage de vestiges sont sauvegardés, y compris des rails et une locomotive, des machines en tant que sculptures etc., puisque leur coexistence avec des bâtiments évoque le passé (Photo 3 et 4 ). Il faut évoquer qu'en Chine la valeur patrimoniale réside principalement dans les aspects économiques liés au tourisme et par conséquent les sites patrimoniaux sont plutôt exploités pour les attractions touristiques. Ensuite, la valeur artistique est appréciée et amplifiée par la valeur économique sur le marché de l'art et la mise en tourisme des sites patrimoniaux. En revanche, la valeur historique n'est présente que dans la recherche, dont la reconnaissance est assez limitée à cause de son statut méconnu en tant que source de recherche. 
Photo ${ }^{\circ} 3$. Ancienne conserverie rouge à Guangzhou

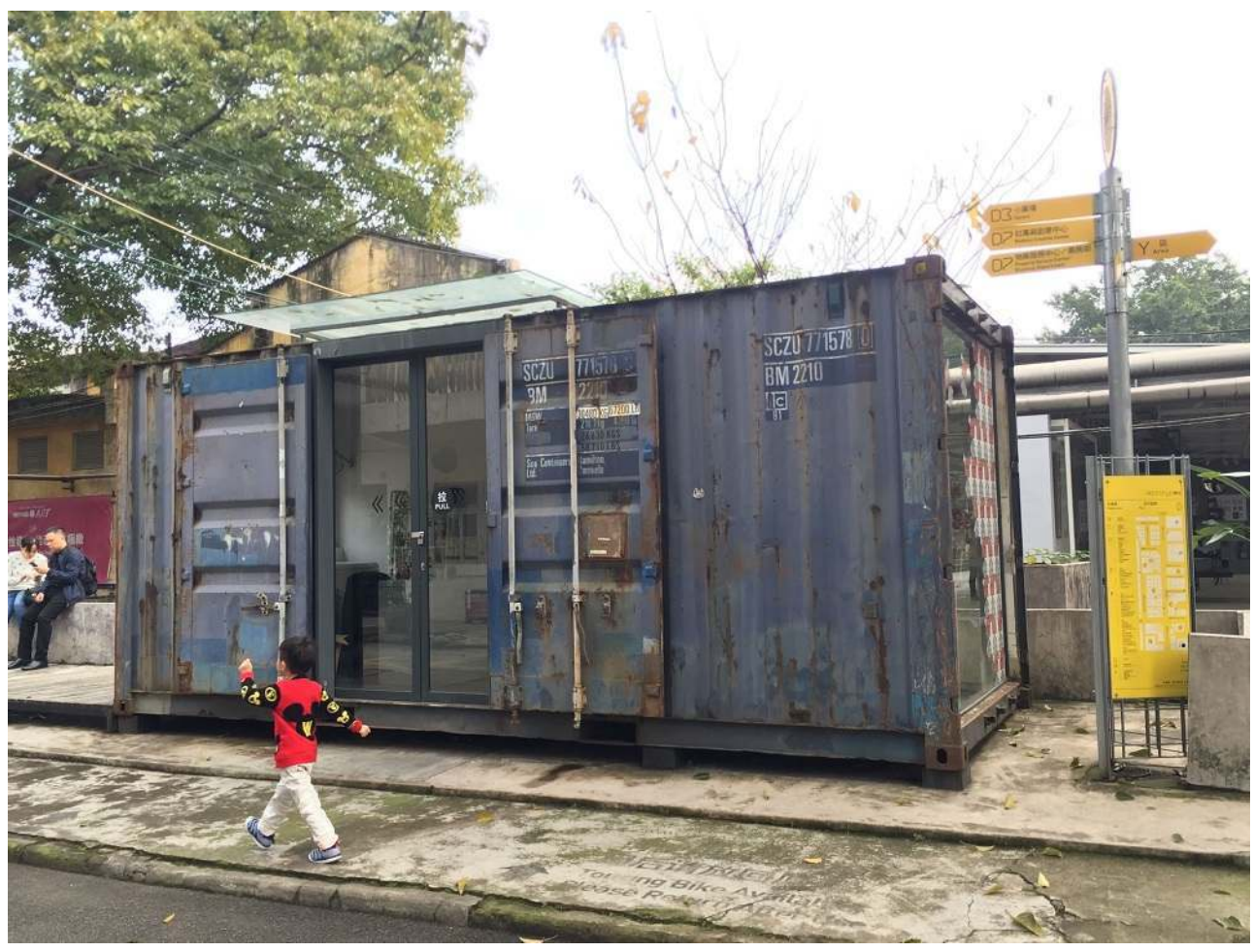

Un conteneur transformé en boutique à côté des voies ferrées.

Crédit personnel de l'auteur 
Photo $\mathrm{n}^{\circ} 4$. Ancienne conserverie rouge à Guangzhou

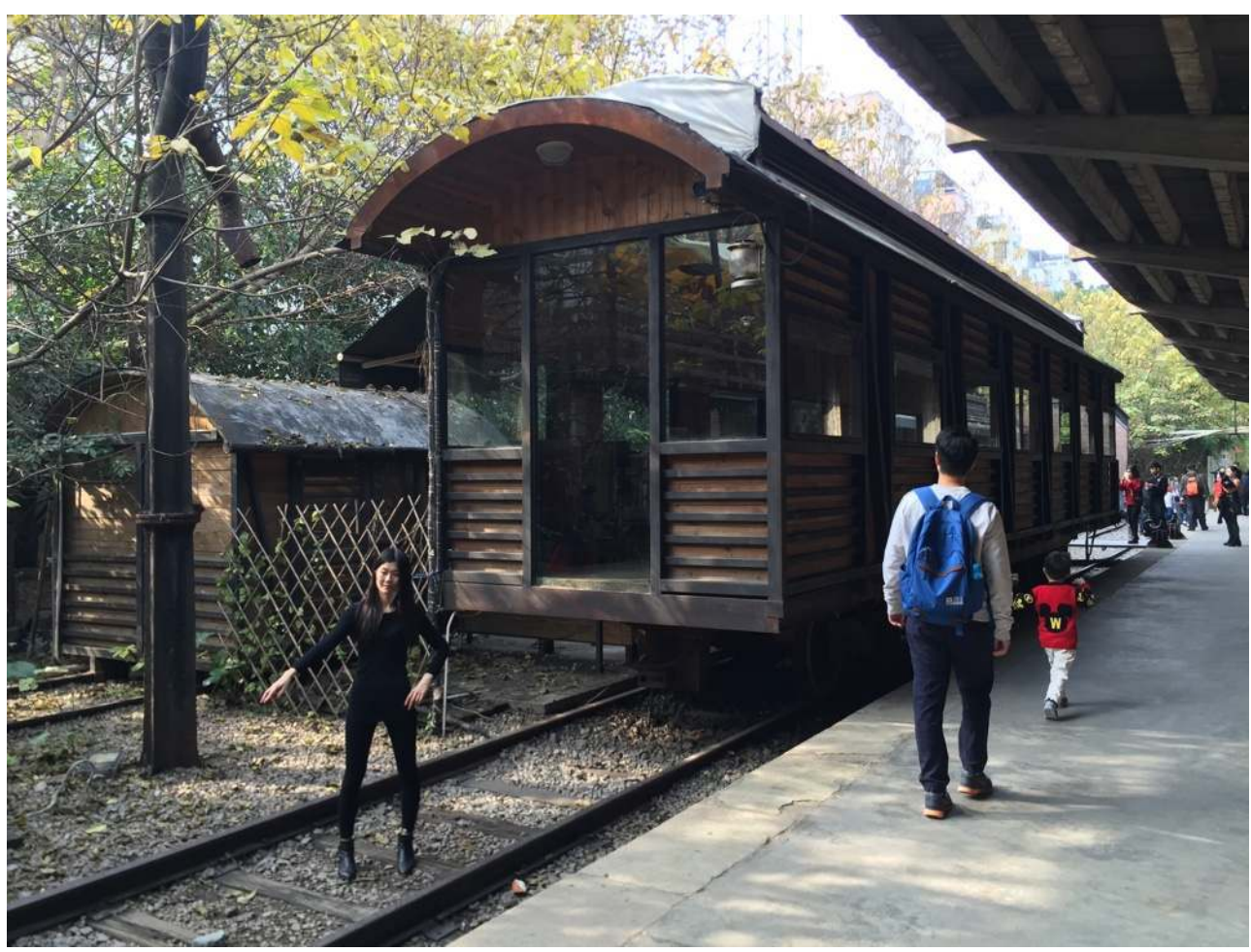

Un wagon ancien préservé comme décoration

Crédit personnel de l'auteur

11 Étant donné que ces terrains sont actuellement une partie intégrante de l'espace urbain, dont la valeur foncière s'élève progressivement, il est probable que de nouvelles fonctions immobilières les remplaceront, si la transformation est médiocre et si les démarches concernant la conservation ne sont toujours pas mises en place'. Nous avons beaucoup déploré le destin de sites importants, qui ont été rasés faute d'une protection justifiée et d'une valorisation des bâtiments historiques. Nous pouvons en citer une: considérée comme une industrie polluante, la Soudière de Tianjin chargée de cent ans d'histoire a dû déménager vers la banlieue de Tanggu. Sa chaîne de production en état intégral est reconnue en tant qu'innovation conçue par les Chinois, et les experts ont recommandé sa conservation à titre patrimonial. Malheureusement, sans attendre l'inscription sur la liste des sites importants, la chaîne a été complètement détruite et le site a été englobé dans le projet d'un quartier financier. De ces faits, on déduit que des avantages de financement sont donnés aux terrains et aux projets plus promoteurs pour un gain économique/ immobilier que ceux qui répondent mieux aux besoins ou ceux qui mériteraient une conservation. En tant que propriétaire des biens culturels, si l'État chinois se préoccupe plutôt de réalisations appuyées par des chiffres impressionnants, au lieu de s'acquitter de l'obligation de préservation pour de véritables enjeux, ce sera une grande perte pour la sociétét ${ }^{10}$.

\section{Cadre juridique}

12 Pour protéger et gérer des biens culturels, il existe deux systèmes parallèles : le Code du Patrimoine et le système de Villes et Villages Exceptionnels d'Histoire et de Culture (VVEHC). 
Le premier divise les biens culturels en deux catégories, hiérarchisées en nationale, municipale et communale : Patrimoine d'importance exceptionnelle (PIE) et Patrimoine d'importance ordinaire (PIO). L'autre système tient à valoriser des villes et villages détenant des quartiers ou des vestiges de grande valeur en termes d'histoire et de culture. Le premier système est géré par le ministère des biens culturels de l'État et ses bureaux locaux, qui sont responsables de la conservation et la restauration des biens immobiliers ainsi que des objets, tandis que le deuxième l'est conjointement par le ministère de la construction et la planification et le ministère des biens culturels.

Il n'est pas surprenant que le patrimoine industriel soit dédaigné en Chine, parce que les deux systèmes ne donnent pas de définition spécifique à cette catégorie. Le seul document le concernant est la Recommandation de Wuxi sur la préservation du patrimoine industriel, parue en 2006, mais elle n'impose cependant pas d'obligations. En second lieu, la protection doit catégoriquement s'appuyer sur une liste complète des biens culturels à l'échelle nationale établie sous la forme d'une base de données recensant les aspects essentiels du bien. Toutefois, très peu de sites industriels étaient inscrits sur la liste du patrimoine important. Il faudra attendre la troisième enquête nationale sur les biens culturels, effectuée de 2007 à 2011, pour qu'un certain nombre de vestiges industriels soient classés au patrimoine ${ }^{11}$. À part ce genre d'enquête entreprise irrégulièrement, il n'existe pas de démarche pour classer un bien culturel, même si les règlements du VVEHC (2008) stipulent que l'autorité est responsable de l'enregistrement des sites d'une valeur patrimoniale. Par conséquent, nous sommes incapables de réagir devant des cas urgents quand un site non-classé est confronté à un risque de dommage ou de démolition. En troisième lieu, à cause de leur statut d'infériorité par rapport au patrimoine classique, les budgets pour la restauration et l'entretien des bâtiments industriels sont très limités. De plus, il n'existe pas de mécanisme juridique permettant le recours à d'autres ressources financières, telles que les fondations et les donations. Enfin, un grand décalage, tant temporel que géographique, demeure en termes de standards juridiques dès le début de l'exécution du programme VVEHC, qui mérite une analyse plus approfondie.

Ce système qui existe depuis 1982, n'a pas encore été appliqué, puisqu'aucune loi n'a été mise en place pour imposer des obligations et des normes. Pourtant, chaque ville reconnue par le système élabore son propre cadre juridique pour guider les pratiques. Guangzhou a adopté ses règlements de VVEHC en 1999 pour permettre d'engager des démarches administratives concrètes ; Tianjin s'est dotée de règlements pour la sauvegarde des architectures stylistiques en 2005, afin de préserver son héritage architectural unique ; Shanghai a intégré dans la planification urbaine des mesures pour désigner les quartiers historiques et assurer la sauvegarde des bâtiments en tant que repères historiques. Il a fallu attendre 2008 pour que les règlements au niveau national soient promulgués. En termes d'exécution, les règlements stipulent que la ville ou le village inscrit VVEHC doit mettre en place une planification de protection des quartiers historiques et culturels qui soit cohérente avec l'aménagement urbain ; une liste des bâtiments importants avec des critères de contrôle et avec l'estimation de la durée des projets, doit également être précisée. En réponse, la ville de Guangzhou a réalisé un enregistrement des bâtiments importants, dont une partie des sites industriels et des habitats vernaculaires, sous le label bâtiments historiques et stylistiques, un troisième label qui n'assure aucune protection juridique. 
Outre la diversité des conditions selon les villes, les règlements nationaux ont deux défauts, si on les compare avec le système français et anglais. D'un côté, il n'existe pas de critères universels et ils ne sont pas bien définis en ce qui concerne le contrôle de la zone autour d'un bien immobilier, tandis qu'en France il s'agit d'un rayon de 500 mètres. Ce contrôle est effectivement le principe essentiel pour valider des projets urbains en cours et à venir, qui pourraient constituer des menaces sur les biens. Ainsi, aucune ambiguïté ne doit exister quand il s'agit d'obligations. D'autre part, l'objectif du système, qui considère les quartiers en tant qu'ensemble, est un bon point de départ pour maintenir l'identité de la ville. Néanmoins, du fait que ces règlements ne tiennent pas compte des bâtiments individuels en général, le destin de chaque bâtiment en dehors de la protection de PIE ou PIO n'est pas résolu. Par contre, pour la France et pour l'Angleterre, la protection d'un bien est précisée jusqu'aux composants du bâtiment ou du site, de sorte qu'on puisse intervenir librement sur les structures en respectant les règlements. Nous pouvons ainsi en déduire que le système juridique nous sert de principe pour faire évoluer l'environnement urbain et les architectures anciennes selon nos besoins, ce qui est favorable pour le patrimoine. L'ambiguïté et les standards différents ne font qu'empêcher ce processus.

\section{La recherche académique}

La recherche sur le patrimoine constitue une force considérable pour justifier, promouvoir et piloter la conservation du patrimoine, que ce soit pour révéler ses valeurs représentatives, ou pour améliorer les méthodes scientifiques de classement, de gestion et de valorisation. D'abord, la notion et la perception du patrimoine sont essentielles pour nous aider à définir ce qui est le patrimoine spécifique du pays et du peuple. Malheureusement, les vestiges industriels en Chine sont un domaine patrimonial mal défini, mal connu et mal protégé. Cette méconnaissance du patrimoine industriel tient à la jeunesse de l'industrie par rapport à la longue histoire de la Chine, et au fait que l'accent est mis plutôt sur les sites monumentaux que sur ceux qui représentent l'époque industrielle. Si on remonte aux origines du patrimoine industriel, c'est l'archéologie industrielle qui cherche à décrypter les gigantesques structures et machines liées à ce passé. En France comme en Angleterre, la volonté d'éclairer l'histoire du développement de certaines industries consiste à rechercher les différentes modalités de développement et comprendre leurs liens avec les différents acteurs à l'aide de vestiges physiques. Ces efforts font finalement avancer la connaissance sur les atouts et faiblesses de leurs systèmes, et en particulier sur les moyens conçus et les compétences acquises par l'homme, afin de s'en servir et au besoin de les améliorer.

En Chine, la situation est différente. Le patrimoine est un concept tout nouveau emprunté au milieu international, mais les Chinois ont une attitude plutôt pragmatique envers le patrimoine, qui est pour eux un nouveau vecteur économique lié au tourisme. Sa valeur pour faire avancer la recherche et transmettre la connaissance du passé est toujours méprisée. Inévitablement, les chercheurs en architecture et urbanisme, et ceux qui s'intéressent au tourisme sont plus intéressés par les sites et constructions physiques que les historiens. Il n'est pas étonnant qu'en tant que connaissance d'un lieu patrimonial, nous ne trouvions qu'un discours historique succinct sur la date de construction, le parcours de développement avec quelques anecdotes sur des personnages connus ou sur la fabrique. La raison principale en est que ces chercheurs ne sont pas capables d'avoir 
une notion intégrale du patrimoine. Quant au patrimoine industriel, les vestiges comprennent non seulement l'architecture, mais aussi la machinerie, les archives, et le paysage naturel et artificiel, tel que la forêt, les ports et les mines, etc. Ses liens physiques et immatériels avec le territoire sont présents d'une façon organique, et cela demande de la part des chercheurs de développer des compétences pour déchiffrer ces liens relatifs à la localité. La méthode centrée sur l'architecture nous éloigne des archives, qui fournissent pourtant des informations quantitatives et qualitatives sur l'architecture, précieuses mais non visibles. Même en matière d'architecture, les sites construits uniquement avec un objectif de production se distinguent nettement des architectures religieuses ou classiques, et les structures industrielles différentes méritent chacune une réflexion et une interprétation nuancées. Si ces traces physiques ne sont pas valorisées comme elles doivent l'être, il reste peu d'espoir de conserver des informations contenues dans la mémoire orale de la communauté ouvrière. Cette génération nous échappera dans quelques décennies et la lacune d'informations sur l'industrie locale rendra floue l'identité de la ville. Bien que la Chine commence à reconnaitre le patrimoine immatériel et s'efforce d'inscrire sur la liste mondiale du patrimoine immatériel des formes telles que l'artisanat, les coutumes, les arts du spectacle etc., celles-ci se limitent à l'époque ancienne à cause de la disparition rapide des praticiens. Toutefois, les savoir-faire de l'époque industrielle à l'instar des connaissances, des conceptions, et des gestes, disparaissent si les acteurs praticiens ne laissent aucune trace. Les Chinois ne s'intéressent quasiment pas à cette forme du patrimoine immatériel parce qu'il est peu appréciable en termes esthétiques.

La façon de classer et de valoriser le patrimoine repose sur l'étude des valeurs historiques, artistiques et techniques de sites spécifiques. On peut aussi constater qu'en Chine, la méthode dominante de classification des industries modernes se fait en fonction de la nature de la propriété et non en fonction du domaine industriel, plus précisément en entreprises nationales et entreprises investies par des étrangers. Ainsi, le classement du patrimoine suit le même chemin, ce qui fait qu'on a coupé les liens entre les entreprises d'un même type d'industrie ${ }^{12}$. Au contraire, une recherche comparative sur ces deux catégories complémentaires est indicative, dans le sens où les compagnies de différente nature mais du même secteur industriel ont connu des situations différentes. On est capable de déterminer quels sont les impacts connus sur cette industrie, tandis que d'autres impacts ne touchent que des entreprises d'une dimension ou d'une propriété d'une autre nature. À travers la recherche thématique sur la technique et ses hybridations $s^{13}$ nous mettrons en relation des particularités, et nous éclairerons des échanges au niveau individuel et synthétiserons la tendance générale, qui n'est pas si évidente. De plus, le défaut de recherche thématique par industrie entrave l'efficacité du classement des sites les plus représentatifs, alors que le classement est le principe de valorisation des sites éminents dans le cadre du Patrimoine Mondial. À mon avis, la représentativité est un outil utile pour mieux répartir des ressources limitées à travers des choix raisonnables. Ensuite, il nous paraît dommageable que les aspects humains n'occupent pas une place importante dans le patrimoine de la recherche conventionnelle, alors que ce sont des architectes qui ont conçu les bâtiments, des ingénieurs qui ont introduit l'innovation et la perfection des procédés entraînant des changements radicaux. La présence physique et psychologique de l'homme dans son environnement de travail est cruciale: des échecs et des réussites, des conditions défavorables et des apprentissages maîtrisés, des négociations paisibles et des grèves conflictuelles se juxtaposent. Au fur et à mesure, on s'est rendu compte que les interactions entre 
l'homme et l'environnement, et entre les différents acteurs donnent naissance à des institutions et à des instruments plus sophistiqués, tels que la corporation et la législation, qui en retour assurent efficience et justice. Ces indications offertes par le patrimoine industriel et dignes d'une découverte plus approfondie nourriront la connaissance du passé et l'enthousiasme pour ces vestiges industriels.

\section{Des tournants et de nouvelles pistes}

Étant donné la richesse des aspects d'un site ou d'une structure industrielle, la recherche patrimoniale requiert des collaborations transdisciplinaires comprenant l'identification, l'étude historique, le constat d'état, le recensement, la conservation et la valorisation appropriée à la localité sur le long terme. Afin de réutiliser les bâtiments industriels obsolètes mais susceptibles d'être réadaptés, une réhabilitation et des restaurations seront nécessaires. Une réhabilitation vise à rééquiper un bâtiment avec des dispositifs conformes aux usages futurs, tels que la sécurité contre l'incendie, la climatisation et le chauffage, l'éclairage etc. selon les fonctions prévues pour le bâtiment. Une restauration sera indispensable pour remettre en état et consolider certaines structures précaires ou manquantes en vue d'un usage à long terme. Dès la conception d'un projet de réhabilitation, il faudra tenir compte des aspects associés développés ci-dessus, en visant un développement durable dans l'avenir. Ce sont les enjeux du patrimoine industriel pour la société moderne, en tant que ressources et repères culturels, que nous espérons gagner. Pour un pays aussi déséquilibré que la Chine, il faut que les trois milieux se soutiennent et se coordonnent pour piloter les pratiques dans la bonne direction. Nous avons constaté une grande ouverture d'esprit aujourd'hui, grâce aux échanges interculturels forts et fréquents, et aux tournants marquants et positifs pris par ces trois milieux. Nous les illustrerons, et ensuite proposerons des pistes pour équilibrer les enjeux économiques, sociaux et ceux de la conservation du patrimoine.

20 À Shanghai, la douzième planification quinquennale a fait des progrès significatifs par rapport à la onzième, en insistant sur les principes d'intervention à l'égard des bâtiments anciens. Ces principes mettent l'accent sur l'état constant d'un bien immobilier: la propriété, la structure architecturale et la nature foncière. À Guangzhou, la nouvelle planification de l'aménagement urbain fait appel à une valorisation des bâtiments contemporains dont des bâtiments industriels, et cette démarche nécessite évidemment une connaissance complète des sites sous forme d'une liste complémentaire. Cette tendance de la réglementation plus précise et rigoureuse en ce qui concerne le patrimoine démontre que l'État se rend compte de la manière pertinente de gérer une richesse en cours, à la fois reconnue et menacée. Dans l'avenir, cela nécessitera une série de règlements plus inclusifs en vue de classer et de hiérarchiser les bâtiments modernes en fonction de leur importance, en leur donnant un titre justifié et une position équivalente à celle des architectures anciennes et classiques. Dans la pratique concrète, une base de données nous semble indispensable, regroupant toutes les informations concernant le bien, y compris son histoire et ses valeurs, son état physique, sa propriété et sa protection juridique, les interventions effectuées, les impacts environnementaux et l'usage futur de ce bien. Récemment, les autorités en charge de biens culturels ont décidé de publier la liste complète des biens classés, dont l'accès est cependant réservé aux officiels et spécialistes du patrimoine. Une telle base de données devra servir de point de départ et d'instrument commun de gestion des biens pour les entités intéressées par le 
patrimoine, telles que les universités, les institutions patrimoniales, les bureaux d'urbanisme, les agences d'architectes et le public. Cet outil facilitera la mise à jour des informations, leur partage et circulation immédiats, le tri des renseignements selon différents critères, et la prise de décision à tous les niveaux, que ce soit pour des cas urgents ou pour des contrôles sur des interventions ${ }^{14}$. En donnant un accès libre et en garantissant les droits réservés de manipulation, cet outil performant contribuera à donner de fructueux résultats pour la recherche, la conservation et les décisions politiques.

21 Il faut aussi souligner que des architectes chinois ont réalisé de bonnes reconversions telles que le Parc de Qijiang à Zhongshan, qui était autrefois un chantier-naval, et le Musée d'art contemporain à Shanghai, auparavant centrale électrique et salle provisoire de l'Exposition Universelle de Shanghai en 2008. En outre, nous ne citerons pas les nombreuses tentatives de reconversion de bâtiments démodés, qui ont surgi pour des usages sportif, gastronomique et d'enseignement. Ce mouvement de mise en valeur des bâtiments d'environ cinquante ans indique que la quantité de bâtiments démodés d'une importance ordinaire augmente, et que ceux-ci s'apprêtent à répondre à nos besoins multiples. Quand on projette la désaffection des friches industrielles, il faudra être plus courageux et ambitieux et donner de l'importance à la culture en tant que lien avec la localité et levier du développement local, au lieu de se limiter à une créativité considérée comme haut de gamme. Nous avons constaté des demandes de la part du public pour apprécier le paysage industriel et se divertir dans ces bâtiments, se confronter à son passé et s'imprégner de la réflexion nostalgique sans oublier à quel point nous nous situons dans l'histoire. Certes, l'industrialisation permet au début d'atteindre des objectifs économiques, mais son développement sans mesure produit des effets destructifs et entraine la perte des enjeux identitaires à long terme. Il vaut mieux concevoir des projets de transformations progressives et transitionnelles en recréant l'élan économique et en impliquant la communauté locale. Le moyen d'impliquer le public consiste à transformer des friches industrielles en espaces destinés à des activités culturelles variées pour enrichir leur vie, et promouvoir toute une chaîne de production « culturelle » : le théâtre, les expositions, la danse, les foires et la mode. Sans nul doute, un profit économique prometteur suivra, qui permettra au site de regagner une force productive et la multifonctionnalité antérieures, et à la communauté locale d'obtenir des bénéfices économiques.

22 À l'instar de la Ruhr en Allemagne et de Pantin à Paris, la culture est considérée comme la clé et la communauté est mise au cœur de ces projets, qui respectent l'histoire locale et injectent des éléments novateurs en faveur de l'évolution de la localité. Par exemple, la transformation du complexe de l'ancienne mine à Essen a été réalisée en deux décennies, et ce lieu abrite aujourd'hui une grande variété d'éléments culturels : le Musée de la Ruhr, le Musée de Red Dot Design, l'École de design, des bureaux, des restaurants, un hôtel, une piscine, une patinoire, un hall de spectacles, des espaces pour des concerts, et des itinéraires pour les cyclistes et promeneurs. À Pantin, des manufactures et bâtiments anciens ont été réhabilités pour devenir des bureaux, un centre de danse, une galerie d'art ; le quartier a bénéficié de la réimplantation d'ateliers et d'instituts de recherche des grandes marques de la mode et il continue à évoluer. Dans la Ruhr, le sol et l'air contaminés ont disparu et le quartier vétuste et délabré de Pantin a renouvelé son image. En présentant des expériences réussies, nous préconisons des projets réalisés étape par étape, de sorte que des modifications soient possibles si des obstacles et des changements 
de situation se présentent. De même, des investissements modestes connaîtront probablement une rentabilité rapide par rapport à un projet global très coûteux au départ.

Les dernières recherches sur le patrimoine industriel s'élargissent à de nouvelles pistes. Le projet mené par la Faculté d'Architecture de l'Université de Tianjin porte sur le système d'évaluation et de classement du patrimoine industriel fondé sur les valeurs, qui est adapté et opérationnel en Chine. Dans ce cadre, des recherches ont révélé des structures d'une valeur exceptionnelle en démontrant des aspects historiques liés à l'évolution du site et des éléments techniques liés aux fonctions productives. En plus, le recours à l'exploitation des archives est considéré comme un moyen complémentaire à l'étude des preuves physiques. L'enquête sur les sites industriels d'un territoire selon la distribution géographique et le domaine industriel fait l'objet d'une seconde piste de recherche. Ces recherches ont pour vocation d'illustrer le processus d'industrialisation, ses caractéristiques ainsi que les milieux technique et social d'un lieu donné. Quand il s'agit de la conservation, de nouvelles recherches prennent en compte le bien et son environnement dans son intégralité, ce qui est particulièrement juste dans les cas où l'industrie a façonné le tissu urbain des cités ouvrières ou des infrastructures telles que les chemins de fer, les ports etc. Donc, l'authenticité d'un site industriel s'incarne non seulement dans ses éléments architecturaux, mais aussi dans ses liens matériels et immatériels avec son environnement et son milieu social. On remarque une tendance à la création d'associations spécialisées dans le patrimoine industriel en Chine en faveur d'une coopération étendue. La Commission Académique du Patrimoine Industriel de l'Association d'Architecture a annoncé en 2010 dès son inauguration l'Initiative de Pékin, ayant pour vocation la sauvegarde du patrimoine industriel. Cette commission organise une réunion par an à l'occasion de laquelle des chercheurs présentent leurs recherches sur des sujets thématiques, qui feront l'objet d'une publication. Ces recherches constituent de nouveaux jalons dans ce domaine en associant les valeurs historiques à la valorisation, et en réfléchissant sur les enjeux liés à l'actualité et sur la conservation préventive. En ce qui concerne la recherche sur la pratique, les discussions ne manquent pas sur la faisabilité des projets reposant sur l'estimation des effets socio-économiques, sur la capacité d'accueil du site, ainsi que sur la pertinence des mutations. La Commission du Patrimoine Industriel de l'Association des Biens Culturels, établie en 2014, met l'accent sur la création de plateformes pour le partage d'informations concernant la conservation et la valorisation.

24 La formation des spécialistes dans le domaine de la conservation patrimoniale est partagée entre deux acteurs principaux: les universités dotées de département de patrimoine culturel, ou de muséologie, ou d'archéologie d'un côté, et l'Institut de recherche sur le patrimoine culturel ${ }^{15}$, l'organisme officiel dépendant du Bureau des biens culturels de l'autre. Dans le premier cas, les étudiants sont formés selon leur matière choisie parmi une grande variété (histoire de l'art, gestion de bases de données, techniques de restauration). Dans le deuxième, l'institut est responsable de l'organisation des formations pour le bureau, en collaboration avec d'autres établissements nationaux et étrangers. Il existe des modalités diversifiées de cours thématiques, séminaires, visites de terrain, et la plupart se focalisent sur la restauration et les techniques tandis que peu de séances sont consacrées à la conservation des sites patrimoniaux. Le troisième acteur responsable de la formation est l'ICOMOS Chine, qui organise les concours d'architectes et d'agences qualifiés pour les projets de restauration sur les sites patrimoniaux. Ces acteurs 
tendent à collaborer pour la formation des spécialistes et des étudiants universitaires à travers la création d'un diplôme coordonné. Il n'y pas encore des formations spécialement dédiées au patrimoine industriel sauf dans les études architecturales pour une orientation praticable. Nous espérons que l'archéologie industrielle et l'histoire des techniques se développeront en Chine pour former des spécialistes capables de révéler l'histoire des sites et de s'engager dans leur valorisation.

\section{Conclusion}

Après avoir retracé cette histoire de la valorisation du patrimoine industriel en Chine avec l'étude des modalités des parcs créatifs, nous pouvons affirmer que le patrimoine industriel constitue un véritable enjeu pour l'aménagement urbain, la revitalisation industrielle, ainsi que la maintenance de l'identité de la ville dont les urbanistes, les conservateurs du patrimoine et les chercheurs se préoccupent. Le patrimoine évolue comme les autres tissus urbains, mais il détient une signification symbolique liée au passé qui contribue à enrichir nos racines culturelles. Pour des villes ou des quartiers industriels, qui sont démunis d'histoire glorieuse et ne doivent leur naissance qu'à l'industrialisation, l'industrie constitue à la fois leur histoire et leur culture. Il ne faudra pas tout conserver comme il ne faudra pas tout détruire. La seule façon de parvenir à un équilibre satisfaisant est de trouver la bonne méthode pour identifier les vestiges essentiels et valoriser ses particularités en termes d'histoire et de culture, afin qu'ils puissent être reconnus, appréciés et utilisés. La fascination qu'exerce le patrimoine industriel tient à l'affinité qu'il entretient avec notre génération par rapport à l'héritage classique, et à son potentiel énorme qui offre un champ d'expérimentation pour nos plus grandes idées et notre imagination en matière d'architecture. En Chine, la perte des tissus urbains traditionnels et la similarité des villes sont le prix payé dans la première phase de l'expansion urbaine caractérisée par la rapidité et le désordre. Dans la deuxième phase, où l'on est confronté à la désaffectation des friches industrielles, c'est la qualité de vie et les liens organiques qui comptent. Avec les avantages que représentent l'ouverture d'académies, les privilèges donnés par les politiques et le marché croissant de la création culturelle, il faudra s'appuyer sur la connaissance fondamentale de ce genre de patrimoine, des critères concrets et rigoureux pour sa conservation, ainsi que sur les besoins de la société pour prendre des décisions aussi prudentes que prospectives.

\section{BIBLIOGRAPHIE}

BAILEY Christopher, MILES Steven, STARK Peter, « Cultural-led Urban Regeneration and the Revitalisation of Identities in Newcastle, Gateshead, and the North East of England », International Journal of Cultural Policy, 10 (1), p. 47-65.

CUNNINGHAM Stuart, « From Cultural to Creative Industries: Theory, Industry and Policy Implications », Media Internatioanl Australia, 102, 1, 2002, p. 54-65. 
DENG Yawen, Étude sur le développement des parcs créatifs de Guangzhou sous l'angle de la réutilisation du patrimoine industriel, Guangzhou, Université d'industrie de Guangdong, 2013.

FAUCHER Hélène, Les enjeux de la conservation et de la réhabilitation du patrimoine industriel, dimension juridique et prospective, http://www.patrimoineindustriel-apic.com/N/i22.html. HARTLEY John (Ed.), Creative Industries, Wiley-Blackwell, January 2005.

« Heritage Inventories, Conservation Perspectives », The GCI Newsletter, Fall 2013.

JI Hong, XX Subin, NOBUO Aoki, «Identification et classification des valeurs scientifiques et techniques du patrimoine industriel : étude de cas sur le patrimoine industriel à Tianjin ", Nouvelle Architecture, février 2012, p. 28-33.

JI Hong, XU Subin, NOBUO Aoki, «Étude historique et évaluation du patrimoine industriel : étude de cas sur le Chantier-naval de Daku de marine du nord », Revue d'Architecture, 2011, p. 80-85.

JI Hong, «L'évolution de la distribution des industries modernes à Tianjin et l'état actuel de la sa conservation comme patrimoine ", Revue de l'Université de Fuzhou, 42, 3, 2014, p. 439-444.

JOOST Dessein (Ed.), Culture in, for and as Sustainable Development, Conclusions from the COST Action IS1007 Investigating Cultural Sustainability, University of Jyväskylä, 2015.

LIU Boying, LI Kuang, « Les trois époques importantes du développement industriel en Chine », La planification et construction de Pékin, 1, 2011, p.8-12.

LIU Hui, LIU Huadong, « Identification et système de conservation du patrimoine industriel à Guangzhou », Urbanisme et Architecture, 10, 2015, p. 32-35.

LÜ Liang, La régénération des quartiers historiques par l'introduction de l'industrie créative :étude de cas sur le M50 de Shanghai, Shanghai, Université Tongji, 2006.

PENG Qi, Étude sur la planification des parcs créatifs à Guangzhou, Guangzhou, Université de technologie de Chine méridionale, 2016.

RIGAUD Jean-Luc, Les magasins généraux de Pantin, Histoires de mutations, 2015.

XU Jian, SUN Qinyi, L'ambiance créative et la stratégie de planification dans la désaffectation des quartiers démodés : étude de cas de la réhabilitation de quatre usines à Guangzhou, Recueil de la Réunion sur l'urbanisme de Chine, 2013.

YAN Mi, NUBUO Aoki, XU Subin, La gradation de la préservation patrimoniale de la Soudière de Tianjin, de la méthode de valeur patrimoniale, Constructions Industrielles, 45, 5, 2015, p. 34-37.

ZHENG Ying, ZHANG Wei, Réflexions sur la conservation du patrimoine moderne non-classé de Tianjin, Nouvelle Architecture, 2012, 4, p. 49-53.

\section{Sources et webographie}

Code du Patrimoine de Chine.

Le règlement des villes et villages exceptionnels d'histoire et de culture.

Ministère de la Culture et de la Communication : Les Monuments historiques, mode d'emploi, 2004.

Department for Cultural Media \& Sport : Scheduled Monuments \& nationally important nonscheduled monuments, 2013.

Department for Cultural Media \& Sport : Principles of Selection for Listing Buildings, 2010. 
Principles for the Recording of Monuments, Groups of Buildings and Sites (1996), ICOMOS.

Centre des industries de Shanghai : http://www.scic.sh.cn.

Ville de Pantin : http://www.ville-pantin.fr/grandsprojets-zac_du_port.html.

Department for Cultural Media \& Sport, Scheduled Monuments \& nationally important nonscheduled monuments, 2013.

Department for Cultural Media \& Sport, Principles of Selection for Listing Buildings, 2010.

Principles for the Recording of Monuments, Groups of Buildings and Sites (1996), ICOMOS.

Centre des industries de Shanghai : http://www.scic.sh.cn.

Ville de Pantin : http://www.ville-pantin.fr/grandsprojets-zac_du_port.html.

\section{NOTES}

1. L'industrialisation en Chine est souvent divisée en trois périodes avant l'instauration de la république: 1840-1894, naissance des industries; 1895-1936, investissements étrangers et nationalistes ; 1937-1949, guerres sino-japonaise et intérieure. Le développement industriel après l'instauration de la république se divise en quatre périodes : 1949-1952, rétablissement après la guerre; 1953-1963, développement avec les aides soviétiques; 1964-1978, développement autonome ; 1979 à nos jours économie de marché et commerce international.

2. Ce document est issu d'une collaboration entre l'ICOMOS Chine, l'ICOMOS Australie et le Getty Institute et il est le guide le plus détaillé en matière de conservation du patrimoine culturel. Il a été actualisé en 2014 pour s'adapter aux changements dans les secteurs qui ont un impact sur la conservation patrimoniale.

3. Ce mouvement s'interprète en deux sens: la restructuration des industries et le réaménagement des terrains urbains. Il fut propulsé par la politique du même nom, annoncé par le gouvernement à l'orée du XXIe siècle, qui visait à déplacer les industries lourdes et polluantes vers la banlieue des grandes villes et à transformer ces terrains pour accueillir des services tertiaires plus appréciés et adaptés au mode de vie métropolitain. La restructuration signifie des changements radicaux tels que la fermeture, le regroupement et le réinvestissement des équipements, renforçant éventuellement la compétitivité. Limitées par la pression de la croissance démographique et de la congestion, ces grandes villes bénéficieront des espaces et de la main d'œuvre libérés par cet appel.

4. Cette planification urbaine de Guangzhou est vouée spécifiquement à libérer des espaces industriels et transformer des bâtiments obsolètes en leur attribuant de nouvelles fonctions, y compris plus de 200 projets de réhabilitation. L'importance de la transformation des vieux bâtiments au lieu de les démolir est soulignée.

5. CUNNINGHAM Stuart, From Cultural to Creative Industries: Theory, Industry and Policy Implications, Media International Australia, 102, 1, 2002, p. 54-65; HARTLEY John (Ed.), Creative Industries, Wiley-Blackwell, January 2005

6. En 2009, l'État annonça les principes de planification et promotion de l'industrie culturelle, qui engendra des politiques plus concrètes au niveau local. Dans le document sur les avis de revitalisation de l'industrie culturelle et créative et les services de design, communiqué en 2014, l'État met l'accent plus explicitement sur la construction, la direction et les avantages en termes de soutien financier pour les parcs créatifs. En 2015, le chiffre estimé de parcs créatifs dépasse 2000 à l'échelle nationale, dont 336 sont dénommés modèles et 10 sont d'importance nationale.

7. Nong Tang est un tissu urbain typique à Shanghai caractérisé par des ruelles longues et étroites sur les deux côtés dans lesquelles se trouvent des maisons qui s'ouvrent sur la ruelle par 
une petite porte et qui donnent à l'intérieur accès à une cour plus large accueillant plusieurs pièces de la maison.

8. DENG Yawen, Étude sur le développement des parcs créatifs de Guangzhou sous l'angle de la réutilisation du patrimoine industriel, Chapitre 4,107p.

9. Le site de Xingfang 60 à Guangzhou était l'usine d'équipements mécaniques, qui a été transformée en parc créatif servant de bureaux pour de petites entreprises en 2008. Il n'avait que cinq ans de fonctionnement quand il a été détruit et remplacé par un gratte-ciel.

10. Dans tous les rapports et documents officiels, les objectifs et les résultats ne se mesurent que par des chiffres. Par exemple, la réussite des parcs créatifs en 2013 de Pékin s'exprime en 742 entités de service, 140,78 milliards, 148000 employés etc. Quant à la planification de la construction de zones pour l'industrie culturelle et créative (2014-2020), les critères sont aussi quantifiés : « 6 pointus » signifiant les six quartiers dédiés aux services pointus ; « 4 nouveaux » signifiant quatre nouveaux quartiers etc.

11. Il faut préciser que les enquêtes menées en 1996, 2001 et 2006 ont classé 14, 28 et 44 sites industriels en tant que PIE, dont une vingtaine relèvent de l'époque moderne. En 2013, ce nombre est passé à 329 , dont 84 relèvent de l'époque moderne.

12. Le nouveau mode de classification apparait dans ces articles : JI Hong, XX Subin, NOBUO Aoki, Identification et classification des valeurs scientifiques et techniques du patrimoine industriel : étude de cas sur le patrimoine industriel à Tianjin, Nouvelle Architecture, février 2012, p. 28-33.

13. Une hybridation technique se réfère à une forme technique combinant deux variétés ou caractéristiques techniques d'origines différentes, soit en termes géographiques soit en termes fonctionnels pour créer une fonctionnalité nouvelle afin de s'adapter aux situations propres.

14. MYERS David, AVRAMIDES Yiannis, DALGITY Alison, "Changing the Heritage Inventory Paradigm: The Arches Open Source System, Heritage Inventories, Conservation Perspectives", The GCI Newsletter, Fall 2013.

15. La mission de cet institut est d'inventorier les biens patrimoniaux, de faire de la recherche, de rédiger les lois et les règlements, de promouvoir la formation et de faciliter les échanges.

\section{RÉSUMÉS}

L'industrialisation de la Chine s'est accélérée depuis la fin du $\mathrm{XX}^{\mathrm{e}}$ siècle, et elle constitue un moteur puissant de l'urbanisation. Dans les années quatre-vingt dix, une nouvelle politique d'urbanisation et d'économie a ciblé les usines polluantes, les installations industrielles démodées et celles jugées non compatibles avec le développement durable. Dans les grandes villes chinoises, beaucoup de ces friches industrielles qui semblaient condamnées à la démolition, on été reconverties en parc créatifs. Cela a d'abord été le fait d'initiatives individuelles, puis c'est devenu une politique à l'échelle nationale, au motif que cela offrait une solution pour la requalification des quartiers urbains délaissés et la conservation du patrimoine industriel. Cette forme de valorisation patrimoniale l'a emporté, de sorte que les autres possibilités ont été négligées. De ce fait, beaucoup de sites industriels, en tant que patrimoine, ont perdu leur valeur d'authenticité. Nous examinerons le statut juridique du patrimoine industriel en Chine, et les modalités de labellisation pour sa conservation, ainsi que les attentes sur son rôle dans le développement économique, afin de comprendre le déséquilibre entre la réutilisation et la protection patrimoniale. Nous proposerons ensuite de nouvelles pistes de recherche académique 
et des modalités de reconversion autres que parc créatif pour définir une approche pertinente de conservation et de valorisation du patrimoine industriel en Chine.

The industrialisation in China accelerated from the end of the $20^{\text {th }}$ century and has always been an impetus of urbanization today. In the nineties, a new urban and economic politics has targeted the polluting factories, and the obsolete industrial constructions, which are incompatible with sustainable development. In the big cities in China, a lot of brownfields are threatened by destruction. Their transformation into creative zones, which began with individual initiatives, has turned into a nation-wide politics. In fact, this way offers us a solution to urban regeneration of the marginalized zones and at the same time to heritage conservation. However, people tend prioritize this practice as the way of giving value to industrial sites so that other possibilities have been neglected. As too many industrials sites have been turned into creative zones and undergone inappropriate alterations, they have lost their value of authenticity as heritage. We try to review the legislation system of industrial heritage, which recognizes its importance and justifies its conservation, and the expectations of its reuse in the context of economic development. This can help us understand the imbalanced situation between heritage reuse and conservation. We also aim to present new horizons in academic research and conversions other than the creative zones, in order to define an appropriate approach of conservation and reuse of industrial heritage in China.

\section{INDEX}

Mots-clés : histoire des techniques, Chine, XXIe siècle, patrimoine industriel, friches industrielles, reconversion

Index chronologique : Époque contemporaine

Index géographique : Chine

Keywords : history of technology, China, 21th century, industrial heritage, brownfields, conversion, creative zones

Thèmes : Horizons internationaux

\section{AUTEUR}

\section{JIALI HUANG}

Jiali Huang est doctorante en Histoire des Techniques sous la direction d'Anne-Françoise Garçon. Intitulée « Les réseaux électriques de Tianjin (1900-1960) : histoire et valorisation patrimoniale », sa recherche interroge l'électrification de la ville de Tianjin en Chine, initiée dans les concessions étrangères de la ville puis transformée et développée par les Chinois, en analysant l'interaction entre les différentes cultures techniques. Elle étudie également les possibilités de valorisation du réseau électrique en tant que patrimoine industriel dans un contexte de multiplicité patrimoniale et d'urbanisation « destructrice». 